\title{
Uso de la estimación de costos como herramienta de evaluación
}

Fecha de recepción:2020-07-15 • Fecha de aceptación: 2020-08-07 • Fecha de publicación: 2020-10-10

\author{
Alison Ibeth López Vargas \\ TAXCONTABLE Cia Ltda, Ecuador \\ lalibeth13@hotmail.com \\ https://orcid.org/0000-0003-2803-6614
}

\section{Resumen}

La globalización, los sistemas tecnológicos, políticas tributarias, entre otros, obligan a establecer estrategias, tales como las herramientas de costos, para determinar cuánto les cuesta producir un servicio. La presente investigación usó como caso de estudio una empresa de transporte y tuvo como objetivo identificar y calcular el costo en la prestación de su asistencia de una manera técnica. Se aplicó un estudio exploratorio-descriptivo y se realizó una encuesta conformada por 6 preguntas de selección múltiple. Los resultados expresaron que el $80 \%$ de encuestados manifiesta que no existe un modelo de cálculo de costos, el $60 \%$ desconoce cómo calcularlos y el $40 \%$ no sabe qué elementos forman parte del costo del transporte. Estos datos fueron analizados e interpretados por el método estadístico. La propuesta establece que el método de costeo fijo y variable es una opción para determinar de manera oportuna el valor de un servicio, facilitando la toma de decisiones a los propietarios de transportes que ofertan su asistencia.

\section{PALABRAS CLAVES: servicio de transporte, costos, cálculo de costos, fijo, variable}

\begin{abstract}
Globalization, technological systems, tax policies, among others, force to implement strategies such as cost tools to determine how much it costs them to produce a service. The present research used a
\end{abstract}


transportation company as a case study and aimed to identify and calculate the cost in providing the assistance in a technical way. An exploratory-descriptive study was applied and a validated survey that was made up of 3 multiple-choice questions was applied to the administrative area. The results expressed that $80 \%$ of respondents state that there is no cost calculation model, $60 \%$ do not know how calculate them and $40 \%$ do not know which elements are part of the cost of transport. These data were analyzed and interpreted by the statistical method. The proposal establishes that the fixed and variable costing method is an option to determine the value of a service in a timely manner, facilitating decision-making by transport owners who offer their services. 


\section{Introducción}

El transporte en la actualidad es considerado una de las necesidades básicas de la sociedad, ya que este promueve el dinamismo comercial y económico de los países. De Rus (2003) manifiesta que la conceptualización de transporte hace referencia a toda actividad que conlleva el transporte y movilización de un bien o personas de un lugar a otro; además de la logística del vehículo también se debe considerar otros aspectos tales como la seguridad del pasajero o la mercancía, y velar por la integridad del medio de transporte (pág. 45).

Hoy en día, tener un control adecuado en los procedimientos, la utilización de herramientas eficientes y la búsqueda de estrategias, permiten un mejoramiento continuo en la administración, la espera al terminar un proceso de compra o prestación de servicios impide al administrador o gerencia que se tome decisiones acertadas en cuanto a los precios de venta y otras decisiones que requieren de una información más oportuna.

Durante su existencia, la empresa caso de estudio, ha obtenido un incremento considerable de contratos de trabajo con instituciones escolares y entidades públicas y privadas que permite la generación de nuevos empleos, existiendo la necesidad de controles para llevar a cabo estrategias administrativas que ayuden a controlar y gestionar sus recursos de manera eficiente, es por esto que se ha tomado como caso de estudio para la presente investigación.

Dentro de los servicios ofertados se encuentran: transporte corporativo, transporte a escuelas y colegios y buses contratados a sitios turísticos. La empresa cuenta con un promedio de 25 años de experiencia y su misión parte de satisfacer las necesidades de las personas mediante su servicio eficiente, seguro y confortable, esto ha permitido plantearse una visión de consolidarse como una empresa confiable, eficiente y rentable en el sector del transporte en el Ecuador.

Para tener un mayor entendimiento es necesario definir ciertos términos que estaremos manejando durante el desarrollo del artículo.

El autor Lawrence (1943) define la contabilidad de costos como "un procedimiento ordenado de uso de los principios de la contabilidad general, para registrar los gastos de explotación de una empresa, de tal manera que las cuentas que llevan en relación con la producción y la venta puedan servir a los directores para la determinación de los costos de producción y de distribución, unitarios o totales, de alguno o de todos los productos manufacturados o servicios prestados y de las diversas funciones de la empresa, para lograr así una explotación económica, eficiente y lucrativa".

Por su parte, Polimeni, Fabozzi, Adelberg, y Kole (1997) dicen que "es una fase ampliada de la contabilidad general o financiera de una entidad industrial o comercial, que rápidamente proporciona a la gerencia datos relacionados con los costos de producción o venta de cada artículo o brindar un servicio en particular".

A esto se suma Toro López (2010), quien sostiene que esta mide, consolida y reporta información financiera relacionada con la adquisición y / o consumo de materiales, brindando información para 
contabilidad administrativa y contabilidad financiera.

Estos autores coinciden que la contabilidad de costos proporciona información útil sobre los costos de producir bienes que permiten a los directivos tomar decisiones. A lo que Aguirre (2004) explica que los valores prudentes y razonables de todos los conceptos y recursos necesarios para desarrollar una actividad se conocen como "costos de un bien o servicio", éstos se clasifican como elementos del costo (pág. 22).

\section{Elementos del costo de producción}

Existen tres elementos para el costo de producción. La materia prima representa el elemento de vital importancia que la empresa utiliza para la elaboración de bienes. La mano de obra es la fuerza física o mental invertida en la transformación o procesamiento de la materia prima de un producto en proceso o terminado. El costo de la mano de obra es el valor que se paga por el uso de los recursos humanos, la mano de obra se conoce como el esfuerzo físico y mental que se pone en un servicio de fabricación. El concepto también se utiliza para nombrar el costo de este trabajo, es decir, el precio pagado. Debido a la inconsistencia de la palabra "obra", la definición se refiere a "obra a mano o con mano", lo que tiene más sentido si decimos que las obras u obras son realizadas por personas (García, 2012).

Y los costos indirectos de fabricación, conocidos también como CIF, son todos los costos que intervienen en el proceso de producción pero no directamente, tales como: materia prima indirecta (hilo, botones de cierre, etc.) mano de obra indirecta (salario de supervisores, salario de contador, salario de gerente-propietario), otros costos indirectos tales como: alimentos de los trabajadores, seguro de fábrica, impuestos de fábrica, energía eléctrica de fábrica, depreciación de maquinaria y equipo de fábrica, depreciación de edificios de fábrica, etc.

Por otra parte, el propósito de los sistemas de costos es acumular los costos de productos o servicios. La información que brindan estos sistemas es utilizada por los gerentes para fijar los precios de sus productos, controlar sus existencias, aplicar acciones correctivas a sus procesos y básicamente saber cuánto cuesta producir un bien o un servicio.

Según cómo se acumulan los costos para pagar la producción, los sistemas de costos se clasifican en: sistemas de costos de órdenes de producción, sistemas de costos de procesos, sistemas de costos estándar, sistemas de costos de absorbentes, sistemas de costos histórico, sistema de costo variable o sistema de costo predeterminado. Dependiendo de las necesidades y características de cada empresa, estos sistemas se pueden utilizar o, a su vez, combinados.

A continuación, estaremos definiendo cada uno de estos sistemas:

- Sistema de cálculo de costos de órdenes de producción: proporciona un registro para cada orden de venta. Se caracteriza por el hecho de que cada uno de los costos incurridos en el proceso de producción está claramente identificado y cargado a la orden de producción correspondiente, utilizada por las empresas que producen bajo pedido. Este sistema, tiene algunas características como: se acumulan por lotes, se maneja producción bajo pedidos específicos, no 
se produce normalmente el mismo artículo.

- El sistema de costo por procesos: en este los costos se acumulan en cada proceso o departamento en el que los productos se utilizan para convertirse en productos terminados. Algunas características de los sistemas de costeo por procesos son que se acumulan por departamento y su producción es continua o en serie.

- Sistema de costo estándar: son los que trabajan a partir de los costos calculados antes del proceso de fabricación, para ser comparados con los costos reales con el fin de verificar si lo que se incorpora en la producción se ha utilizado efectivamente para un cierto nivel de producción, y tomar acciones correctivas (Polimeni et al., 1997). El sistema de costeo estándar es tan estricto que requiere muchos estudios para determinar el coste de producción (Cashin \& Polimen, 1980).

- Sistemas de costeo absorbente: son los que consideran y acumulan todos los costos de producción, tanto costos fijos, como costos variables, siendo considerados como fragmento del valor de los productos elaborados, bajo el indicio que todos los costos son necesarios para fabricar un producto (Moreno, 2002).

- Sistema de costeo histórico: es aquel que acumula costos de producción reales, es decir, costos realizados o incurridos; lo cual puede realizarse en cada una las órdenes de trabajo o en cada una de las fases del proceso productivo.

- Sistemas de costeo por variables: independientemente de qué se fabrique, los costos fijos representan la capacidad de producir y comercializar bienes o servicios y los costos variables dependen del volumen de producción (García-Pérez-de-Lema et al., 2006).

- Sistemas de costeo absorbente: son los que consideran y acumulan todos los costos de producción, tanto costos fijos, como costos variables, siendo considerados como fragmento del valor de los productos elaborados, bajo el indicio que todos los costos son necesarios para fabricar un producto (Moreno, 2002).

- Sistemas de costeo predeterminados: estos son los que trabajan a partir de los costos calculados antes del proceso de fabricación para ser comparados con los costos reales con el fin de verificar si lo que se incorpora en la producción se ha utilizado efectivamente para un cierto nivel de producción, y tomar acciones correctivas (Moreno, 2002).

- Sistemas de costos de calidad: cuantifican financieramente los costos de calidad de la organización agrupados en costos de cumplimiento y no cumplimiento, con el fin de facilitar la selección de la gerencia de niveles de calidad que minimicen sus costos que minimicen sus costos (Moreno, 2002).

A su vez, también existen unas técnicas para determinar costos, estas son el costeo por objetivo, que parte de un precio objetivo y un nivel de beneficio planificado que determinan los costes que debe soportar la empresa para ofrecer dicho producto (coste objetivo = precio objetivo - beneficio deseado). De esta forma, tratamos de ofrecer un producto de calidad, brindamos satisfacción 
en las necesidades del cliente y también ofrecemos un precio razonable ante la demanda. Por otra parte, tenemos la técnica de costeo Kaizen, esta propone actividades para la mejora de las actividades y la reducción de los costos, incluyendo cambios en la forma en que la empresa fabrica sus productos, esto se hace proyectando los costos de las mejoras propuestas, que debe realizarse como un control presupuestario.

Y otros sistemas que también deben considerarse son el de cálculo de costos backflus, este es un sistema de contabilidad de costos condensado en el que los costos de producción incurridos al pasar de una fase a otra no se registran, pero los costos incurridos en los productos se registran cuando se finalizan y/o venden (Gayle, 1999).

Como un sistema alternativo a los tradicionales, aparece el Costeo $\mathrm{ABC}$, el cual identifica las distintas actividades que componen los procesos y los vincula con los productos o servicios en base a su consumo. Este sistema parte de la diferencia entre costos directos e indirectos, vinculando estos últimos a las actividades que se desarrollan en la empresa. Las actividades se presentan de tal manera que los costos indirectos aparecen como directos a las actividades, por lo tanto, se transfieren a los productos (objeto de costos), según la cantidad de actividades consumidas por cada objeto de costos. De esta forma, el costo final está compuesto por los costos directos y los costos asociados a determinadas actividades, consideradas como aquellas que agregan valor a los productos.

A continuación, en la Tabla 1 se establece las diferencias de los sistemas de costos tradicionales versus sistemas de costos $A B C$.

Tabla 1.

Diferencia entre Costeo tradicional y Costeo $A B C$

\begin{tabular}{|c|c|}
\hline Costeo tradicional & Costeo ABC \\
\hline Los productos consumen los costos & $\begin{array}{l}\text { Las actividades consumen los costos, los } \\
\text { productos consumen actividades. }\end{array}$ \\
\hline $\begin{array}{l}\text { Asigna los costos indirectos de fabricación } \\
\text { usando como base una medida de volumen. Una } \\
\text { de las más usadas es la de horas hombres }\end{array}$ & $\begin{array}{l}\text { Asigna de los costos indirectos de fabricación } \\
\text { en función de los recursos consumidos por las } \\
\text { actividades }\end{array}$ \\
\hline $\begin{array}{l}\text { Se preocupa de valorizar principalmente los } \\
\text { procesos productivos }\end{array}$ & $\begin{array}{l}\text { Se preocupa valoriza todas las áreas de la } \\
\text { organización }\end{array}$ \\
\hline Valorización de tipo funcional & $\begin{array}{l}\text { Valorización de tipo trasversal y mejoramiento de } \\
\text { los procesos. }\end{array}$ \\
\hline
\end{tabular}

Fuente: elaboración propia

El mayor reto de las empresas hoy en día consiste en la competitividad, razón por la cual los sistemas de costos juegan un papel importante, pues permiten gestionar una empresa (Lavolpe, 2005). En lo que concierne a Ecuador, la Mypes no utiliza o desconoce sistemas de costos que les facilite determinar un costo de servicio como en el caso del sector del transporte, ya que lo realizan de manera empírica (Bayas, 2018). 
Es vital analizar la importancia que tienen los sistemas de costos en las empresas:

Falcón (2015) en "El costo de la producción y su incidencia en la rentabilidad de la empresa Orecao S.A, Cantón, Quevedo, año 2012" concluyó que la empresa Orecao S.A. utilizó un registro de costos históricos, es decir que los costos son registrados conforme se producen sus pagos o provisiones, por lo que no se aplica la principal norma internacional establecida para la producción agrícola como es la NIC 41 "Activos Biológicos".

Por su parte, Palma (2013) investigó sobre la rentabilidad y determinó que, en el ejercicio contable, al registrar los costos generados por ofrecer un producto o servicio, utilicen procedimientos establecidos en el sistema contable; registrándose bajo el régimen correspondiente y no basado en la experiencia como lo explica el autor, lo que tergiversaría los resultados económicos en un negocio, obteniendo resultados equívocos de la rentabilidad en una determinada empresa.

Mientras que Salinas (2012), con su trabajo "Los costos de producción y su efecto en la rentabilidad de la planta fibra de vidrio en Cepolfi Industrial C.A de la ciudad de Ambato" determinó en sus conclusiones que la industria de partes de fibra de vidrio para autobuses y autos se ha convertido en un sector fundamental para la provincia de Tungurahua, generando empleado directo e indirecto sobre toda la ciudad de Ambato; que mediante la propuesta de un sistema de costos de producción, se puede obtener información real sobre los costos y medir la rentabilidad de la planta de fibra de vidrio en la empresa Cepolfi Industrial C.A; y que la inadecuada forma de controlar los costos, ha generado deficiencias administrativas, sobre todo en la parte de fabricación de los productos, los cuales reportan cantidades erróneas de material ocupado en las requisiciones de productos.

Por lo descrito, el presente caso de estudio tiene como objetivo conocer las causas de la inexistencia de un sistema de costos en la empresa de transportes y proponer un método de cálculo que permita anticipadamente conocer la implicación de los elementos de este sistema para la toma de decisiones en cuanto al valor a ofertar por sus servicios al mercado.

\section{Metodología}

El enfoque de la presente investigación fue cuantitativo, de tipo exploratorio-descriptivo. La metodología fue utilizada en un caso de estudio del cual se utilizó los registros contables de la empresa para su análisis, reflejando las variables más comunes en los contratos de transporte. Se tomó como referencia un contrato, específicamente sobre el costo de una furgoneta, lo que permitió proponer un sistema para identificar y clasificar los valores del servicio ofertado.

Adicional se utilizó como técnica el cuestionario, conformado por 6 preguntas con opciones de selección múltiple. Esta encuesta fue previamente validada por la Magister Lorena Muquis, docente y profesional en el área contable tributaria en la Universidad Israel.

La población seleccionada fue de 5 personas del área administrativa: gerente general, gerente logístico, contador y dos asistentes contables. 


\section{Resultados}

En lo que respecta a la existencia de procedimientos para calcular el costo del servicio, el $80 \%$ de encuestados manifiesta que no existe un modelo de cálculo contable para el servicio de transporte (Figura 1); por lo que se puede interpretar que los empleados no han tomado en consideración la distribución de los costos en la empresa, por lo que la ganancia podría estar por debajo de la esperada. Los sistemas de costos son importantes porque proporcionan información para control de gastos y permite analizar los costos con el objetivo de incrementar la eficiencia para alcanzar objetivos empresariales como maximizar utilidades (González, 2017).

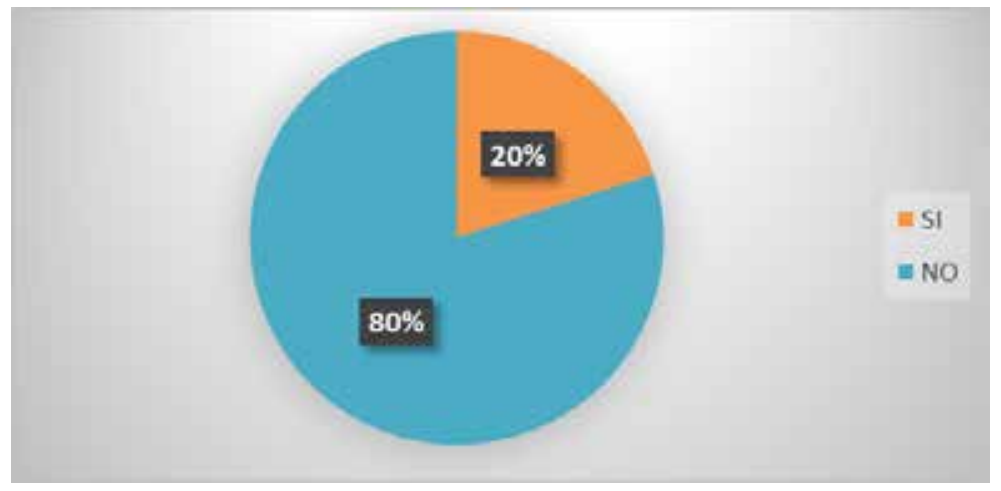

Figura 1. Conocimiento de los empleados sobre la existencia de un sistema de costos

Fuente: elaboración propia

Otro dato que resulta importante mencionar es que el $60 \%$ de los encuestados afirma conocer los rubros que se toman en cuenta para fijar la tarifa del transporte (Figura 2); no obstante, este mismo podría no haberse desarrollado de acuerdo a procesos contables, en vista de que la mayoría no conoce el procedimiento contable de costos. Razón por la cual es importante conocer e identificar los recursos generales utilizados e invertidos, esto hace posible tener información oportuna que permitirá a la administración contar con una mejor visión estratégica diferenciadora de la competencia en cuanto a la toma de decisiones (González, 2017).

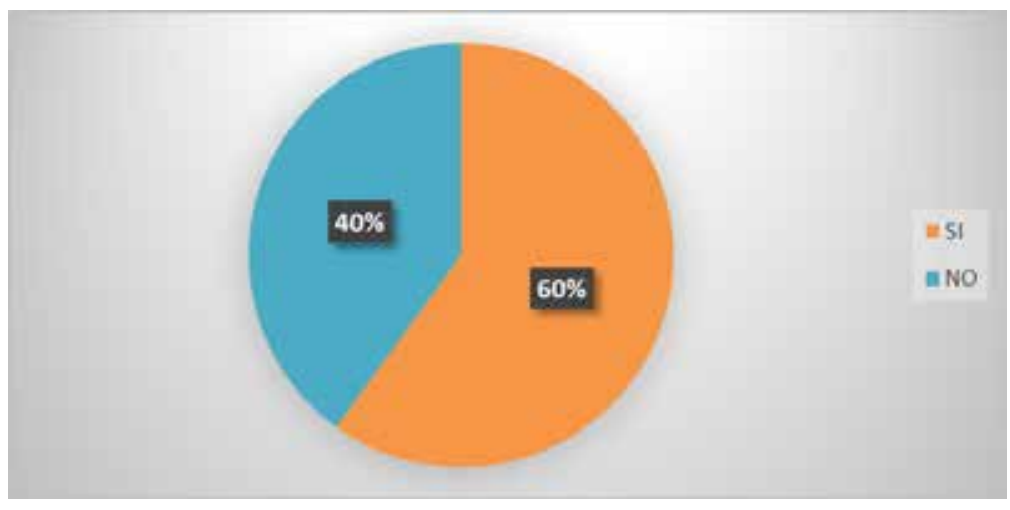

Figura 2. Empleados que conocen los elementos del costo de un servicio

Fuente: elaboración propia 
Adicionalmente, el grupo objetivo manifiesta que contar con sistema de costos permitirá de manera positiva a la administración gestionar de mejor manera los recursos maximizando utilidades, pues el sistema actual no cuenta con parámetros establecidos que permitan un mejor establecimiento en el costo y valor por el servicio. Por lo tanto, se establece que el costeo no se realiza de manera paulatina y no se toman en cuenta costos variables, razón por la cual consideran importante que la administración socialice esta información con todo el personal de la empresa.

\section{Análisis de datos}

En primer lugar, se calculó el valor de mano de obra considerando los beneficios de ley bajo la normativa ecuatoriana establecida en el código de trabajo, para poder determinar el valor de la mano de obra directa se procede a realizar una tabla resumen (Tabla 2) donde se reflejen los sueldos del personal necesario para una sola furgoneta, así como los beneficios sociales a los que los colaboradores tienen derecho según el Instituto de Seguridad Social y el Ministerio del Trabajo.

Tabla 2.

Costo de mano de obra directa

\begin{tabular}{lcccclll}
\hline Representación de Costo de Mano de Obra & & & & \\
\hline Cargo & Sueldo & $\begin{array}{l}\text { Décimo } \\
\text { Tercero }\end{array}$ & $\begin{array}{l}\text { Décimo } \\
\text { Cuarto }\end{array}$ & Vacaciones & $\begin{array}{l}\text { Fondos de } \\
\text { reserva }\end{array}$ & $\begin{array}{l}\text { Aporte } \\
\text { Patronal } \\
\mathbf{1 2 , 1 5 \%}\end{array}$ & Total \\
\hline Chofer & & & & & & \\
& 202,80 & 16,90 & 33,33 & 8,45 & 16,89 & 24,64 & 303,01 \\
\hline Azafata & & & & & & \\
& 133,33 & 11,11 & 33,33 & 5,56 & 11,11 & 16,20 & 210,64 \\
\hline & & & & & & \\
& 336,13 & 28,01 & 66,67 & 14,01 & 28,00 & 40,84 & 513,65 \\
\hline
\end{tabular}

Fuente: elaboración propia

Por otra parte, se realizó el análisis de los costos en base a los datos obtenidos en la empresa, que permitieron identificar los costos en el cual se incurre para brindar el servicio de transporte (Tabla 3). Cabe mencionar que la presente propuesta es una tabla donde se analiza cada costo en los que pueden incurrir los vehículos y los mismos pueden ser cambiados de acuerdo a las necesidades del cliente. 
Tabla 3.

Identificación de elementos del Costo del servicio

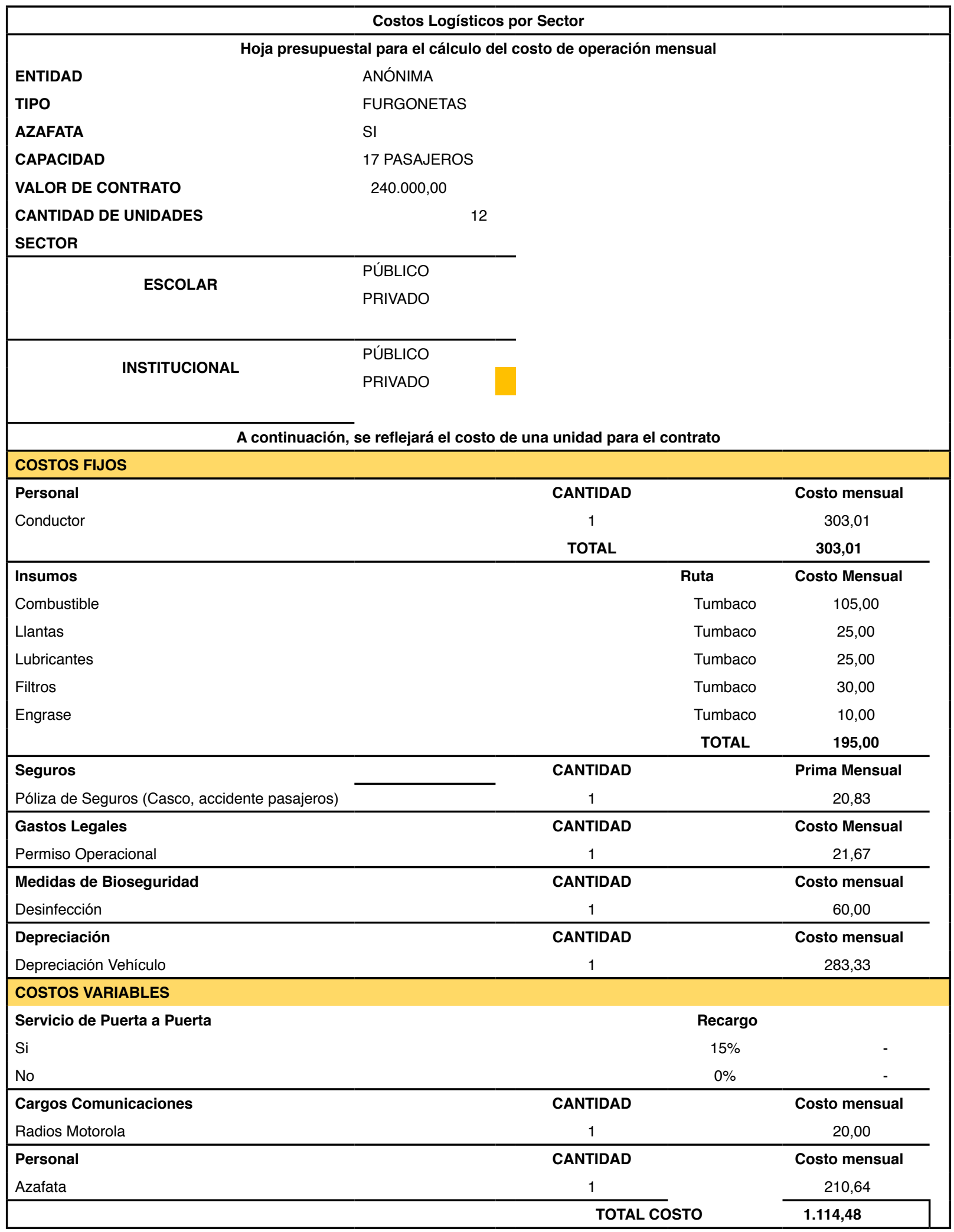

Fuente: elaboración propia 
Una vez finalizado el análisis de los costos con los que incurre el servicio de transporte, tanto en mano de obra, como en costos directos e indirectos, se procede a sumar los valores para conocer cuánto es el costo mensual del servicio: $\$ 1.114,48$.

\section{Conclusiones}

El servicio que ofrece la empresa es de índole privado y público; sustentado la mayor parte de su actividad en el transporte escolar y que al ser un servicio, costea la mano de obra (choferes), costos de mantenimiento y variables según especificaciones del contrato.

La empresa caso de estudio no posee ninguna herramienta para evaluar el uso de los recursos, por lo cual se establece el adecuado cálculo de costo que permita optimizar la gestión. El presente artículo se enfocó en la necesidad de establecer una base que tenga fundamento y no sea instintiva en la prestación de los servicios como se ha venido haciendo en la actualidad, las variaciones de contratos son cotizados únicamente por el gerente, convirtiéndose en una debilidad para la compañía puesto que no existe un sistema de costo que valide el valor de los servicios prestados por parte de la empresa.

Es oportuno resaltar que la contabilidad de costos es un sistema de información cuyos principales objetivos, según Lawrence (1984), son: 1) elaborar informes internos para los gerentes con el fin de ser utilizados tanto en la planificación como en el control de las operaciones del día a día y en la formulación de planes y políticas más amplios; y 2) evaluar inventarios y determinar ingresos, que satisfagan tanto la demanda de información de terceros como de ejecutivos corporativos.

Se determinó además que la compañía no tiene un control contable de los insumos, lo que no les permite desarrollar un adecuado sistema de costeo para su servicio. Se estableció como opción la forma de cálculo "costos fijos y variables", que permite determinar el costo de producción del servicio. 


\section{Referencias}

Aguirre, J. G. (2004). Sistema de costeo. La asignación del costo total a productos o servicios. (1ra edición). Bogotá: Luis Rocca.

Bayas, I. Y. G. (2018). Los sistemas de costos como herramienta fundamental en la administración de empresas. Observatorio de la Economía Latinoamericana, (240).

de Rus Mendoza, G., Campos, J., \& Nombela, G. (2003). Economía del transporte. Antoni Bosch editor.

Falcón, G. (2015). El costo de producción y su incidencia en la rentabilidad de la empresa Orecao S.A, Cantón Quevedo, año 2012 (Tesis de pregrado). Universidad Técnica Estatal de Quevedo, Quevedo, Ecuador.

Gayle, R. L. (1999). Contabilidad y administración de costos.

García, J. (2012). Contabilidad de costos. México.

García Pérez De Lema, D., Marín Hérnandez, S., \& Martínez García, F. J. (2006). La contabilidad de costos y rentabilidad en la Pyme. Contaduría Y Administración, (218), 39-59.

González Delgado, N. D. L. M. (2017). Procedimiento de un sistema de costo. Cofin Habana, 11(2), 91-101.

Lavolpe, A. (2012). Los sistemas de costos y la contabilidad de gestión. Argentina: Consejo Profesional de Ciencias Económicas de la CA de Buenos Aires.

Lawrence, B.W (1943). Contabilidad de costos. Uteha, tomo 1, pag.3.

Lawrence, M. (1984). Estimación de los Costos de Producción.

Moreno, M. M. (2002). Diseño de sistemas de costeo: Fundamentos teóricos. Actualidad Contable Faces, 5(5), 7-22.

Palma, M. (2013). Sistema de costos por procesos y su incidencia en la rentabilidad, de la empresa Soltex, de la ciudad de Ambato, en el primer semestre de 2012 (Tesis de pregrado). Universidad Técnica de Ambato, Ambato, Ecuador.

Polimeni, R. S., Fabozzi, F., Adelberg, A., \& Kole, M. (1994). Contabilidad de costos (No. HF5686. C66 1994.). Bogotá: McGraw-Hill.

Salinas, G. (2012). Los costos de producción y su efecto en la rentabilidad de la planta fibra de vidrio en Cepolfi Industrial C.A de la ciudad de Ambato (Tesis de pregrado). Universidad Técnica de 
Ambato, Ambato, Ecuador.

Toro López, F. J. (2010). Costos ABC Y presupuestos: Herramientas para la productividad. Coleccion: Ciencias administrativas (Colombia). 